\title{
Comparison of Box-Behnken and central composite designs in optimization of fullerene loaded palm-based nano-emulsions for cosmeceutical application
}

\begin{abstract}
Box-Behnken (BBD) and central composite rotatable designs (CCRD) were used as statistical multivariate methods in the formulation optimization of fullerene loaded nano-emulsions. Effect of palm kernel oil ester (10-20\%, w/w), emulsifier (5-10\%, w/w) and xanthan gum $(0.6-1.0 \%, \mathrm{w} / \mathrm{w})$ as formulation variables on the particle size, $\zeta$-potential and viscosity of the nano-emulsions were investigated. Under the optimum conditions, CCRD model predicted the response values for particle size, $\zeta$-potential and viscosity were 153.6. nm, -53.4. $\mathrm{mV}$ and 42.1. Pa. s, respectively. Nonetheless, BBD model suggested that the optimum conditions for a fullerene loaded nano-emulsion would gave particle size, $\zeta$-potential and viscosity of 151.6. $\mathrm{nm},-53.8 . \mathrm{mV}$ and 43.1. Pa. s, respectively. The actual response according to suggested compositions for both models showed excellent agreement with the predicted value with residual standard error (RSE) of less than 4\%. Optimum nano-emulsions were stable during storage at 25 and $45 .{ }^{\circ} \mathrm{C}$ for 90 days and freeze-thaw cycle.
\end{abstract}

Keyword: Nano-emulsions; Fullerene; Response surface methodology (RSM); Central composite rotatable design (CCRD); Box-Behnken design (BBD) 